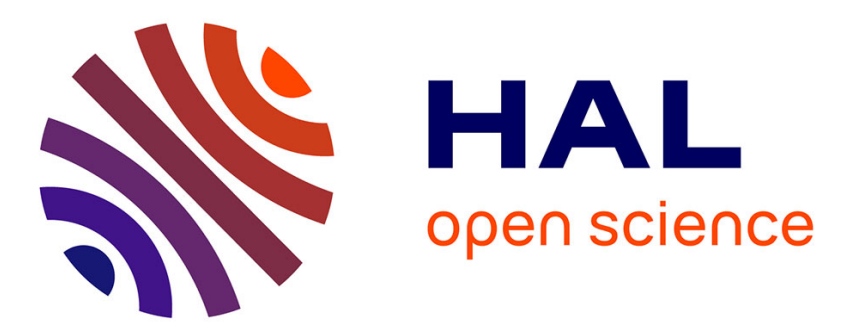

\title{
Platooning of Car-like Vehicles in Urban Environments: An Observer-based Approach Considering Actuator Dynamics and Time delays
}

\author{
Ahmed Khalifa, Olivier Kermorgant, Salvador Dominguez, Philippe Martinet
}

\section{- To cite this version:}

Ahmed Khalifa, Olivier Kermorgant, Salvador Dominguez, Philippe Martinet. Platooning of Car-like Vehicles in Urban Environments: An Observer-based Approach Considering Actuator Dynamics and Time delays. IEEE Transactions on Intelligent Transportation Systems, 2020, 22 (9), pp.5684 - 5696. 10.1109/TITS.2020.2988948 . hal-02545680

\author{
HAL Id: hal-02545680 \\ https://hal.science/hal-02545680
}

Submitted on 17 Apr 2020

HAL is a multi-disciplinary open access archive for the deposit and dissemination of scientific research documents, whether they are published or not. The documents may come from teaching and research institutions in France or abroad, or from public or private research centers.
L'archive ouverte pluridisciplinaire HAL, est destinée au dépôt et à la diffusion de documents scientifiques de niveau recherche, publiés ou non, émanant des établissements d'enseignement et de recherche français ou étrangers, des laboratoires publics ou privés. 


\title{
Platooning of Car-like Vehicles in Urban Environments: An Observer-based Approach Considering Actuator Dynamics and Time delays
}

\author{
Ahmed Khalifa, Olivier Kermorgant, Salvador Dominguez, and Philippe Martinet
}

\begin{abstract}
In this paper, a distributed observer-based approach is proposed to control the longitudinal motion of car-like vehicle platoon moving in an urban environment. To the best of our knowledge, this is the first work presenting an observer-based platoon controller that combines the advantages of high traffic capacity and a minimum number of communication links. To achieve a high traffic flow, a constant-spacing policy is used. However, for that policy, to make platoon string stable, the leader information must be broadcast to all the vehicles. Therefore, we propose a control law in which the predecessor position information is acquired by a sensor-based link while a communicationbased link is used to obtain the leader information. Then, an observer is designed and integrated into the control law such that the velocity information of the predecessor can be estimated without the need to communicate with the preceding vehicle. For navigation in urban environments, we present a third order platoon model represented in the curvilinear coordinates. Conditions for asymptotic stability and string stability are given considering the vehicle actuator dynamics and the induced network/sensor time delay. Finally, we provide both simulation and real-time results to validate our approach feasibility and to corroborate our theoretical findings.
\end{abstract}

Index Terms-platoon in urban environments, curvilinear coordinates, observer-based longitudinal control, limited communication, high traffic flow.

\section{INTRODUCTION}

C AR SHARING system has attracted more attention recently in order to overcome transportation problems. However, cars redistribution among all stations is one of the key issues of such a system. Vehicle platoons guided by professional drivers are proposed in which a leading vehicle, driven by a human, creates and maintains a platoon of vehicles, then navigates inside a city, and finally, redistributes the vehicles over the stations by disassembling the platoon.

This configuration appears as a necessary step towards the use of autonomous vehicles. Indeed, it is now well accepted that autonomous vehicles may follow a different commercial model compared to classical, individual-owned cars. Such vehicles

(C) 2020 IEEE. Personal use of this material is permitted. Permission from IEEE must be obtained for all other uses, in any current or future media, including reprinting/republishing this material for advertising or promotional purposes, creating new collective works, for resale or redistribution to servers or lists, or reuse of any copyrighted component of this work in other works.

The authors are with the Laboratoire des Sciences du Numérique de Nantes (LS2N), Ecole Centrale de Nantes, Nantes, France, given-name. namedec-nantes. fr.

A. Khalifa is on leave from the Department of Industrial Electronics and Control Engineering, Faculty of Electronic Engineering, Menoufia University, Egypt.

P. Martinet is with Inria Sophia Antipolis, Sophia Antipolis, France. can (and should) be used by other people if the previous users have arrived to their destination. Pushing this idea to the limit corresponds to autonomous taxis, that can reach the next customer without a driver. Our framework takes place in an intermediary step, where cars are never fully autonomous: when they are not driven manually (or monitored) by the user, they are part of a platoon in order to be redistributed within the city. Even in this phase, a human operator can take responsibility and monitor the platoon. Urban environments are specific in that the leading vehicle usually moves with varying velocity and along a high curvature path. This makes it difficult to rely on a velocity sensor such as a radar. We have chosen to represent the motion with respect to a path that the vehicle intends to follow, hence the curvilinear/path coordinates are used instead [1]-[3]. Such description allows focusing on the control along the path, and helps coping with high radius of curvature and varying leader velocity. Our proposed methodology to design a platoon control system moving in an urban environment consists of two modules, including longitudinal and lateral controllers. Each of them has an Upper-Level Controller (ULC) and a Lower-Level Controller (LLC). The longitudinal ULC provides the desired vehicle linear acceleration which the car has to follow to achieve the desired longitudinal states while the longitudinal LLC generates the required engine throttle/brake to achieve this desired acceleration. The lateral controller provides the required steering angle to achieve the desired lateral states. In this study, we are interested in designing the longitudinal ULC in the path coordinates with the required transformation to the Cartesian coordinates such that we can design the longitudinal ULC in the path coordinates. The longitudinal platoon control framework depends on four components which are Vehicle Dynamics, Information Flow Topology (IFT), Spacing Policy and Distributed Controller [4]. Different models are used in the literature for the vehicle dynamics including linear and nonlinear models. Some studies directly use nonlinear models for platoon control [5]-[7]. However, these models have difficulty in analyzing system performance against different spacing policies and communications typologies. Three linear models are frequently used. First, a single integrator model is proposed in [8] in which the vehicle speed is taken as the control input but such a model is widely different from the real vehicle dynamics. Second, authors in [9] present a double integrator model that uses the acceleration as the control input but this model ignores some features of vehicle dynamics which may affect the system performance in real-time experiments. Third, 


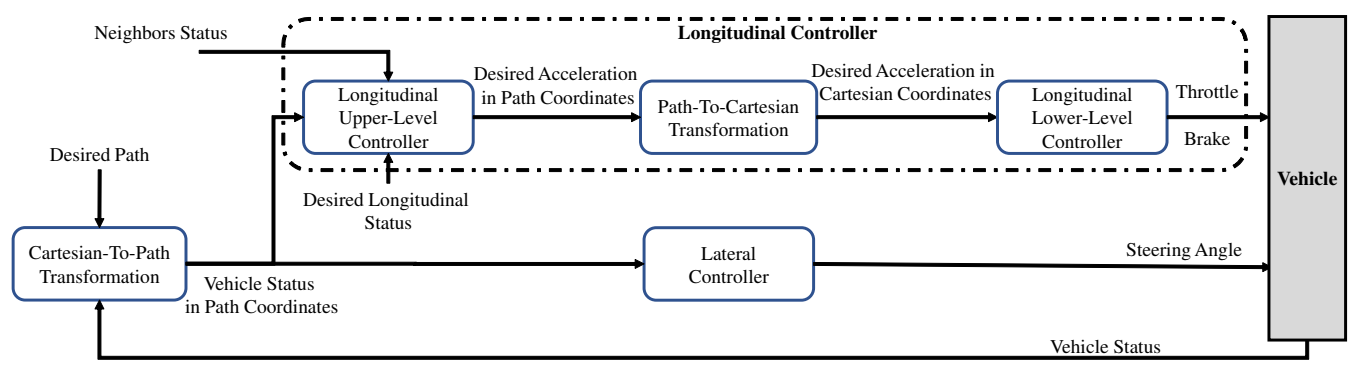

Figure 1. Proposed functional block diagram of a platoon control system navigating in an urban environment.

a third order model is used that considers the input/output behavior of the power-train (i.e., actuator dynamics) [10]. Few studies in the literature have tackled the problem of platooning in the path coordinates, for instance in [1], [11]-[14]. In these studies, the authors assume a first/second order model for the vehicle dynamics that has many defects. Therefore, in this research, a third order longitudinal dynamic model is used and its corresponding one in the path coordinates is presented such that one can design a controller to achieve control objectives in the path (operational) coordinates explicitly. For the distributed control, three main control objectives are required. First, the platoon is able to move in an urban environment. Such an environment can be described by potentially high curvature, varying leader velocity and small inter-vehicle distance, allowing leader-to-follower communication. Second, achieve position and velocity tracking for follower vehicles. Third, guarantee the string stability that ensures that the error signals will attenuate when propagating downstream the vehicle string as the string instability may result in a rear-end collision. In the literature, many platoon controllers are proposed. Adaptive Cruise Control (ACC) is the simplest platoon controller which aims at maintaining a safe distance with the front vehicle. Recently, a distributed version of the platoon controller known as Cooperative Adaptive Cruise Control (CACC) is proposed in which the vehicles use a communication system to share information with their neighbors to improve the whole system functionalities and performance [15]. Six main approaches in designing a platoon controller are proposed, including Linear [16], Optimal [17], $\mathcal{H}_{\infty}$-based [18], Sliding Mode [19], Model predictive [20], [21], and Consensus-based controller [10], [22]-[25]. However, none of these works consider the control in the path coordinates. In our previous work [14], [26], we develop a second-order consensus-based longitudinal controller that considers the conditions of motion in an urban environment (e.g., Curvatures, leader variable velocity, etc.) Several spacing policies are proposed in the literature [27]. The constant distance spacing policy is used commonly due to its capability to achieve very high traffic capacity. However, to achieve string stability, more communication links are required to transmit information of the leading vehicle to all the vehicles in the platoon. The Information Flow Topology describes the inter-vehicle communication topology that the vehicles can use to gather information from its surrounding vehicles. Various IFTs are developed including Predecessor Following (PF), Predecessor-Leader Following (PLF), Bidirectional (BD), etc. The PLF topology is commonly used in the literature in which the leader communicates with all the vehicles in broadcast, and every other vehicle also considers information from its predecessor to compute the control action [27]. However, for a low-cost onboard communication module that may have limited bandwidth capabilities or for a robust communication, high-speed two-channel communication is not feasible or preferred. Therefore, in this study, the proposed control law is designed depending on a hybrid PLF topology. That is, the leader broadcasts its status to all the vehicles by a communication-based link. For the inter-followers information sharing, the inter-vehicle distance is measured by a distance sensor, i.e., by a sensor-based link. For a faster convergence of the platoon, platoon safety, and smooth acceleration of the vehicles motion, and as a result fuel saving and passenger comfort, it is necessary to use the predecessor velocity in the control law. Therefore, we propose an observer-based control technique. A consensus-based controller designed in the path coordinates is the basis of the proposed control strategy and its role is to achieve position and velocity consensus among the platoon members taking into consideration the nature of the motion in an urban environment. An observer is then introduced in the loop to estimate the predecessor velocity. In the literature, several observer-based consensus control algorithms for multi-agent system and platoon system are introduced in [28]-[30] and in [31] respectively. However, these works use the observer to estimate the velocity of either the ego vehicle (i.e., current in-platoon vehicle under control) or the other vehicles based on the communication link among the agents. In addition, they model the agent dynamics as a double integrator that ignore some critical dynamics of the vehicle, e.g. that of vehicle actuator. Moreover, they do not consider the string stability constraint during the controller design and analysis. In our study, the observer does not require any communication link with other vehicles in the platoon which add complexity in the design and analysis in order to guarantee both asymptotic and string stability. The vehicle is modeled as a third order system which considers the actuator dynamics. The main contributions of this paper are given as follows.

- A new third-order longitudinal platoon control framework is proposed considering actuator dynamics and motion in the path coordinates.

- A communication-less observer-based control algorithm is designed such that we can get position and velocity of the predecessor based on onboard distance sensorbased link only without the need for the ego vehicle to 


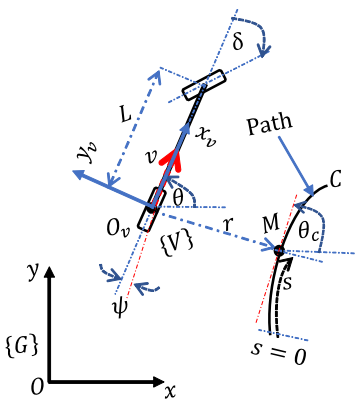

Figure 2. Kinematic model of car-like vehicle.

communicate with the predecessor.

- Under the effect of both network/sensor delays and actuator dynamics, conditions for asymptotic and string stability are provided.

- The proposed approached is validated through realistic simulation and real-time studies.

The paper is structured as follows. Section II formulates the platooning problem given in an urban environment. In Section III, the proposed observer-based control law is presented, and both asymptotic and string stability are analyzed. Simulation and experimental studies are given in Section IV. Section V provides conclusions.

\section{Problem Statement}

\section{A. Car-like Vehicle Kinematic Model}

The kinematic model of the car-like vehicle can be simplified to that of a bicycle model [32], see Fig. 2, in which the left and right wheels are combined into a pair of single wheels at the center of the front and rear axles.

1) Cartesian Coordinates Model: Let $\{V\}, O_{v}-x_{v} y_{v}$, denotes the vehicle body-fixed reference frame with its $x$-axis in the vehicle forward direction and its origin at the center of the rear axle, $O_{v}$, see Fig. 2. The configuration of the vehicle, with respect to the world-fixed inertial reference frame, $\{G\}, O-x y$, is represented by the generalized coordinates $[x, y, \theta]^{T} \in \mathbb{R}^{3}$, where its position is given by $[x, y]^{T}$ and its orientation in the global frame is represented by $\theta . \delta$ is the steering angle in the body frame. The vehicle velocity is $v$ in the vehicle $x_{v}$-direction and zero in the $y_{v}$-direction because the wheels cannot slip sideways. The front wheel is located at distance $L$ from the rear wheel along the orientation of the vehicle. Based on these definitions and configuration, the kinematic model is given by

$$
\begin{aligned}
& \dot{x}=v \cos (\theta), \\
& \dot{y}=v \sin (\theta), \\
& \dot{\theta}=\frac{v}{L} \tan (\delta) .
\end{aligned}
$$

2) Path Coordinates Model: As shown in Fig. 2, the curvilinear coordinates can be represented by $[s, r, \psi]^{T}[3]$, [33]. The tracked path, $C$, defined in the global frame, can be represented as a function of its length $s$ (curvilinear abscissa) at the closest point $M$ to $O_{v}$, the angular deviation, $\psi=\theta-\theta_{c}$, of the vehicle with respect to the path, where $\theta_{c}$ is the path heading at $M$ (in red), and finally, the lateral deviation, $r$, which is the signed orthogonal distance from the center of the rear axle, $O_{v}$, to the closest point on the path, $M$. From the geometry in Fig. 2, the kinematic model in the path coordinates is given by [34]

$$
\begin{aligned}
\dot{s} & =v \frac{\cos (\psi)}{1-r \kappa(s)}, \\
\dot{r} & =v \sin (\psi), \\
\dot{\psi} & =v\left(\frac{\tan (\delta)}{L}-\frac{\kappa(s) \cos (\psi)}{1-r \kappa(s)}\right),
\end{aligned}
$$

where $\kappa(s)$ is the curvature of path at point $M$.

\section{B. Car-like Vehicle Longitudinal Dynamic Model}

Several factors determine the vehicle longitudinal dynamic behavior including the engine, drive line, brake system, aerodynamics drag, tire friction, rolling distance, gravitational force, etc [19]. For the vehicle model, we assume:

Assumption 1. The vehicle body is rigid and symmetric.

Assumption 2. The driving and braking inputs are integrated into one control input.

Assumption 3. The impact of pitch and yaw motions is neglected.

Assumption 4. The power-train dynamics are lumped to be a first-order inertial transfer function.

Therefore, the longitudinal dynamic equation can be represented by

$$
\begin{gathered}
M_{v} a+D_{v} v|v|+G_{v}=F_{v}, \\
\tau \dot{F}_{v}+F_{v}=F_{v, \text { des }}
\end{gathered}
$$

where, $\tau$ is a parameter characterizes the actuator dynamics, $a=\dot{v}$ denotes the vehicle acceleration in the vehicle frame, and $M_{v}, D_{v}, G_{v}$, and $F_{v}$ are the vehicle inertia effect, aerodynamic drag, gravity effect, and input force, respectively.

Input-output linearization [35] is frequently used to eliminate the non-linearities in longitudinal dynamics for the purpose of high-level control design. The inverse dynamicsbased control law is given by:

$$
F_{v, d e s}=M_{v} \mu+D_{v}|v|(2 a \tau+v)+G_{v}
$$

where $\mu$ is the new input signal after system linearization which has to be designed. By applying this lower-level controller, the third order dynamic model can be written in the body coordinates as

$$
\tau \dot{a}+a=\mu .
$$

As we intend to express the controller in the path coordinates (as shown in Fig. 2 ), the relation between the control signals in the path and body coordinates, is recast (2a) as:

$$
\dot{s}=J v,
$$

where $J$ is given by

$$
J=\frac{\cos (\psi)}{(1-r \kappa)} .
$$




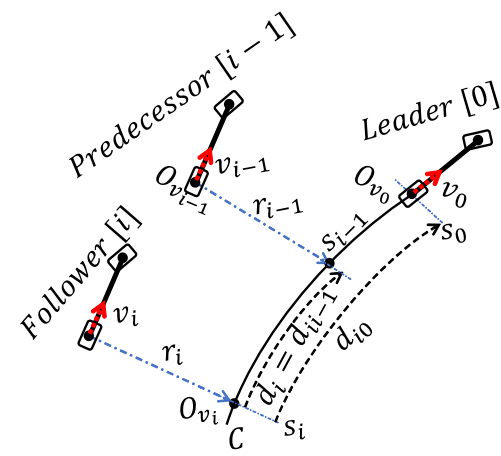

Figure 3. Platoon representation in the path coordinates.

Thus, the vehicle acceleration in the path coordinates, $\eta$, is given by

$$
\eta=J a+\dot{J} v
$$

where $\eta=\dot{q}=\ddot{s}$ and $q=\dot{s}$ are the vehicle acceleration and velocity in the path coordinates respectively. Consequently, if the mapping from the control signal in the path coordinates, $u$, to that in the body coordinates, $\mu$, is given by

$$
\mu=\frac{1}{J}(u-\dot{J} v-2 \tau \dot{J} a-\tau \ddot{J} v),
$$

Note that from (7), $J=0$ when $\psi= \pm \pi / 2$, ie the vehicle orientation is exactly orthogonal to the path tangent at $M$. While this situation is never encountered in practice, a lower threshold on $|J|$ can easily deal with this singularity. The vehicle longitudinal dynamic model in the path coordinates can be represented by:

$$
\begin{aligned}
& \dot{s}=q, \\
& \dot{q}=\eta, \\
& \tau \dot{\eta}+\eta=u .
\end{aligned}
$$

\section{Platoon Longitudinal Model}

Consider $N+1$ vehicles are required to move in a platoon, as illustrated in Fig. 3, including a leading vehicle with index 0 and $N$ following vehicles with index $i ; i=1,2, \ldots, N$. The vehicles can share information by either sensor-based or communication-based link. As it is shown in Fig. 3, the path is not defined beforehand but is built online from the path of the leader vehicle. In practice, all followers receive the position of the leader (typically at $10 \mathrm{~Hz}$ ) and rebuild a continuous path using splines. The vehicle dynamics in the path coordinates can be given by

$$
\begin{aligned}
\dot{s}_{i} & =q_{i}, \\
\dot{q}_{i} & =\eta_{i}, \\
\tau_{i} \dot{\eta}_{i}+\eta_{i} & =u_{i} .
\end{aligned}
$$

where the transformation between the two coordinates can be done by (9).

For the string of vehicles, the inter-vehicle distance, $d_{i}=$ $s_{j}-s_{i}$, is the actual curvilinear distance between vehicle $i$ and its predecessor $j=i-1, d_{i 0}=s_{0}-s_{i}$, is the actual curvilinear distance between vehicle $i$ and leader, $d_{r, i}$ is the desired inter-vehicle distance between vehicles $i$ and $j$, and

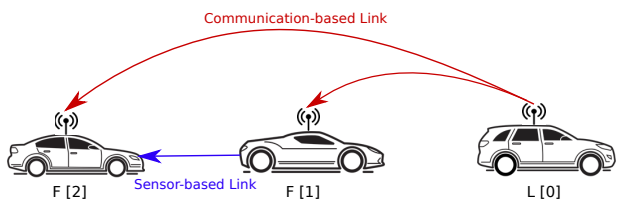

Figure 4. Information flow topology: Hybrid PLF through communication link between leader and followers and direct sensor-based measurements between followers.

$d_{r, i 0}$ is the desired inter-vehicle distance between vehicles $i$ and 0 . For high traffic capacity, the constant spacing policy method is used.

\section{Control Objectives}

The platoon has to move in an urban environment and to track a human-driven leader. The information is shared among vehicles in the form of hybrid PLF topology, see Fig. 4, in which each follower, $i$, can receive the position, velocity, and acceleration of the leader, $s_{0}, q_{0}$ and $\eta_{0}$ respectively, via a communication based-link and position information of the predecessor, $s_{i-1}$, via a sensor-based link. In this work we assume the use of a LiDAR or a camera, which large field of view allows both localization and perception of the predecessor along the curve. On the opposite, it is not accurate enough to estimate the velocity of the precedessor. The communicationbased and the sensor-based information may be affected by time delay. Considering these conditions, $\forall i=1, \ldots, N$, the following goals have to be achieved: first, estimation of the preceding vehicle velocity; second, position and velocity tracking; third, string stability.

\section{Control Design}

This section starts by presenting the proposed observerbased control scheme. Then, the asymptotic platoon stability and string stability analysis are conducted.

\section{A. Observer-based Control Law}

The information flow structure among the platoon can be modeled by a directed graph (digraph) $\mathcal{G}=(\mathcal{V}, \mathcal{E}, \mathcal{M})$ characterized by the set of nodes $\mathcal{V}=1, \ldots, N$ and set of edges $\mathcal{E} \subseteq \mathcal{V} \times \mathcal{V}$. Each vehicle is a node whose dynamics are given by (11). A graph topology is described by an adjacency matrix $\mathcal{M}=\left[m_{i j}\right]_{N \times N}$, in which the element $m_{i j}=1$ if vehicle $i$ can obtain information from vehicle $j$; otherwise, $m_{i j}=0$.The Laplacian matrix $\mathcal{L}=\left[l_{i j}\right] \in \mathbb{R}^{N \times N}$ associated with graph $\mathcal{G}$ is given by $l_{i j}=-m_{i j}$ if $i \neq j$, and $l_{i j}=\sum_{k=1}^{N} m_{i k}$ if $i=j$. From Fig. 4 , in our case $\mathcal{L}$ yields:

$$
\begin{cases}\forall i \in[2, n]: & l_{i, i-1}=-1 \\ \forall i \in[1, n]: & l_{i, i}=1\end{cases}
$$

For the Predecessor - Leader Following (PLF) information flow topology, the elements of the adjacency matrix is given as $m_{i j}=1$ if $j=i-1$, and $m_{i j}=0$ otherwise. The basic PLF-based control law is given by

$$
\begin{aligned}
u_{i}(t)= & g_{c, 3} \eta_{0}+\left(1-g_{c, 3}\right) \eta_{i}(t)+g_{c, 2} e_{q, i 0}\left(t-t_{d}\right) \\
& +g_{c, 1} e_{s, i 0}\left(t-t_{d}\right)+g_{o, 1} e_{s, i}\left(t-t_{d}\right)+g_{o, 2} e_{q, i}\left(t-t_{d}\right),
\end{aligned}
$$


where $e_{s, i 0}(t)=s_{0}(t)-s_{i}(t)-d_{r, i 0}$ is the ego-leader position error, $e_{q, i 0}(t)=q_{0}(t)-q_{i}(t)$ is the ego-leader velocity error, $e_{s, i}(t)=s_{j}(t)-s_{i}(t)-d_{r, i}$ is the ego-predecessor position error, and $e_{q, i}(t)=q_{j}(t)-q_{i}(t)$ is the ego-predecessor velocity error. $G_{c}=\left[g_{c, 1}, g_{c, 2}, g_{c, 3}\right]^{T}$ and $G_{o}=\left[g_{o, 1}, g_{o, 2}\right]^{T}$ are controller parameters to be designed.

The time required for data transmission (ego-leader), sensor-to-controller delay (ego-predecessor), and controller-toactuator delay (neglected) are the sources for the time delays in this system. These different sources of delays can be combined and represented by a single delay. The wireless communication delays has a larger effect on the whole time delay. However, they are mainly affected by the number of vehicles inside the platoon. In addition, it is known that the number of vehicles in a platoon varies on a slow timescale, thus delays can be considered as constant. If needed, online identification of time delays and their dynamics can be performed as shown in [36]. Based on this discussion and for analysis purposes, we can assume:

Assumption 5. $t_{d}$ is the constant upper bound of the system delays.

Remark 1. All the information required to apply this control law (13) is available except the velocity of the predecessor or the velocity error between the ego vehicle, $i$, and the predecessor velocity, $e_{q, i}$, which has to be estimated using an observer.

For the observer design, let us define two states $z_{1, i}=e_{s, i}$ that is known (i.e., measured by the sensor mounted on vehicle $i$ ), and $z_{2, i}=e_{q, i}$ which is unknown and we need to estimate. The state equations that relates these two states are given by

$$
\begin{aligned}
& \dot{z}_{1, i}=z_{2, i}, \\
& \dot{z}_{2, i}=\dot{e}_{q, i} .
\end{aligned}
$$

The state $z_{2, i}$ can be estimated using the following observer

$$
\begin{aligned}
& \dot{\hat{z}}_{1, i}=\hat{z}_{2, i}+h_{1}\left(z_{1, i}-\hat{z}_{1, i}\right), \\
& \dot{\hat{z}}_{2, i}=h_{2}\left(z_{1, i}-\hat{z}_{1, i}\right),
\end{aligned}
$$

where $H=\left[h_{1}, h_{2}\right]^{T}$ is the observer gain.

Exploiting the observer output in the control law (13), then the $i^{t h}$ observer-based control law is proposed as

$$
\begin{aligned}
u_{i}(t)= & g_{c, 3} \eta_{0}+\left(1-g_{c, 3}\right) \eta_{i}(t)+g_{c, 2} e_{q, i 0}\left(t-t_{d}\right) \\
& +g_{c, 1} e_{s, i 0}\left(t-t_{d}\right)+g_{o, 1} \hat{z}_{1, i}(t)+g_{o, 2} \hat{z}_{2, i}(t) .
\end{aligned}
$$

Let us define the error state vector of vehicle $i$ as $f_{i}=$ $\left[f_{1, i}, f_{2, i}, f_{3, i}\right]^{T}$ with $f_{1, i}=e_{s, i 0}, f_{2, i}=e_{q, i 0}$, and $f_{3, i}=$ $e_{\eta, i 0}=\eta_{0}-\eta_{i}$. In order to remove higher-order dynamics, the leading vehicle velocity is assumed to be constant. We will see in the experimental section that our approach is robust to this hypothesis. In this case, the $i^{\text {th }}$ closed-loop state space model is given by

$$
\dot{f}_{i}(t)=A_{f} f_{i}(t)+B_{f} \epsilon_{i}(t)
$$

$$
\begin{aligned}
& \text { where } A_{f}=\left[\begin{array}{lll}
0 & 1 & 0 \\
0 & 0 & 1 \\
0 & 0 & 0
\end{array}\right], B_{f}=\left[\begin{array}{c}
0 \\
0 \\
\frac{1}{\tau_{i}}
\end{array}\right] \text {, and } \\
& \epsilon_{i}(t)=-g_{c, 3} e_{\eta, i 0}(t)-g_{c, 2} e_{q, i 0}\left(t-t_{d}\right) \\
& -g_{c, 1} e_{s, i 0}\left(t-t_{d}\right)-g_{o, 1} \hat{z}_{1, i}(t)-g_{o, 2} \hat{z}_{2, i}(t) \text {. }
\end{aligned}
$$

Consider that $z_{1, i}=e_{s, i}=e_{s, i 0}-e_{s, j 0}$ and $\hat{z}_{i}=\left[\hat{z}_{1, i}, \hat{z}_{2, i}\right]^{T}$, then (15) can be rewritten in concise form as

$\dot{\hat{z}}_{i}(t)=\left(A_{z}-H C_{z}\right) \hat{z}_{i}(t)+H C_{z f}\left(f_{i}\left(t-t_{d}\right)-f_{j}\left(t-t_{d}\right)\right)$,

where $A_{z}=\left[\begin{array}{ll}0 & 1 \\ 0 & 0\end{array}\right], C_{z}=[1,0]$, and $C_{z f}=[1,0,0]$. Defining $G_{c}=K Q_{1}, G_{o}=K Q_{2}, K=\left[k_{1}, k_{2}, k_{3}\right]$, $Q_{1} \in \mathbb{R}^{3 \times 3}$ and $Q_{2} \in \mathbb{R}^{3 \times 2}$, then (18) can be recast as

$$
\epsilon_{i}(t)=-K Q_{1} f_{i}\left(t-t_{d}\right)-K Q_{2} \hat{z}_{i}(t),
$$

Let $F=\left[f_{1}^{T}, \ldots, f_{N}^{T}\right]^{T}, \hat{Z}=\left[\hat{z}_{1}^{T}, \ldots, \hat{z}_{N}^{T}\right]^{T}$ and $\mathcal{E}=$ $\left[\epsilon_{1}, \ldots, \epsilon_{N}\right]^{T}$, after some algebraic manipulations, then the system collective dynamics are given by

$$
\begin{aligned}
\dot{F}(t)= & \left(I_{N} \otimes A_{f}\right) F(t)-\left(I_{N} \otimes B_{f} K Q_{1}\right) F\left(t-t_{d}\right) \\
& -\left(I_{N} \otimes B_{f} K Q_{2}\right) \hat{Z}(t), \\
\dot{\hat{Z}}(t)= & \left(I_{N} \otimes\left(A_{z}-H C_{z}\right)\right) \hat{Z}(t)+\left(\mathcal{L} \otimes H C_{z f}\right) F\left(t-t_{d}\right),
\end{aligned}
$$

where, $I_{N}$ and $\otimes$ denotes $(N \times N)$ identity matrix and Kronecker product respectively.

\section{B. Asymptotic Stability}

Asymptotic stability is a key condition for platoons, and reflects the stability of the platoon as a system [4], [37] ${ }^{1}$. Its analysis typically depends on the considered IFT. In our case, the proposed observer is fully part of the IFT. In the form of time-delayed system, (21) and (22) can be recast as

$$
\dot{X}(t)=A_{o} X(t)+A_{d} X\left(t-t_{d}\right),
$$

where $X(t)=\left[F^{T}(t), Z^{T}(t)\right]^{T}$,

$$
\begin{gathered}
A_{o}=\left[\begin{array}{cc}
I_{N} \otimes A_{f} & -I_{N} \otimes B_{f} K Q_{2} \\
O_{2 N \times 3 N} & I_{N} \otimes\left(A_{z}-H C_{z}\right)
\end{array}\right], \\
A_{d}=\left[\begin{array}{cc}
-I_{N} \otimes B_{f} K Q_{1} & O_{3 N \times 2 N} \\
\mathcal{L} \otimes H C_{z f} & O_{2 N}
\end{array}\right],
\end{gathered}
$$

$O_{N}$ and $O_{N 1 \times N 2}$ denote $(N \times N)$ and $(N 1 \times N 2)$ null matrices respectively.

From Leibniz-Newton formula, we have

$$
X\left(t-t_{d}\right)=X(t)-\int_{-t_{d}}^{0} \dot{X}(t+\rho) d \rho .
$$

Hence, substituting (26) into (23) we get

$\dot{X}(t)=A_{a} X(t)-A_{m} \int_{-t_{d}}^{0} X(t+\rho) d \rho-A_{d}^{2} \int_{-t_{d}}^{0} X\left(t+\rho-t_{d}\right) d \rho$

\footnotetext{
${ }^{1}$ asymptotic stability is referred as internal stability in [4]
} 
where $A_{a}=A_{o}+A_{d}$ and $A_{m}=A_{d} A_{o}$. The matrix $A_{a}$ is given by

$$
A_{a}=\left[\begin{array}{cc}
I_{N} \otimes\left(A_{f}-B_{f} K Q_{1}\right) & -I_{N} \otimes B_{f} K Q_{2} \\
\mathcal{L} \otimes H C_{z f} & I_{N} \otimes\left(A_{z}-H C_{z}\right)
\end{array}\right],
$$

Proposition 1. Assuming $\Gamma \in \mathbb{R}^{2 \times 3}$ satisfies:

$$
\left(A_{z}-H C_{z}\right) \Gamma-\Gamma\left(A_{f}-B_{f} K\right)=-H C_{z f},
$$

then the matrix $A_{a}$ defined in (28) is Hurwitz stable if the following conditions are satisfied:

1) The gain matrices $Q_{1}$ and $Q_{2}$ must satisfy

$$
Q_{1}+Q_{2} \Gamma=I_{3}
$$

2) Constraints on the gain vector $K$ as

$$
k_{2}>\tau_{i} \frac{k_{1}}{k_{3}}, \forall k_{1}, k_{2} \text {, and } k_{3}>0 .
$$

3)

$$
\lambda_{\max }\left\{\Gamma B_{f} K Q_{2}\right\}<\lambda_{\min }\left\{-\left(A_{z}-H C_{z}\right)\right\} .
$$

Proof: For the PLF information flow topology, $\mathcal{L}$ is an lower-triangular matrix and its eigenvalues are all equal to 1 . Therefore, for vehicle $i$, the matrix $A_{a}$ can be represented as

$$
\bar{A}_{a}=\left[\begin{array}{cc}
A_{f}-B_{f} K Q_{1} & -B_{f} K Q_{2} \\
H C_{z f} & A_{z}-H C_{z}
\end{array}\right] .
$$

Exploiting the similarity transformation technique by multiplying the left and right sides of the matrix $\bar{A}_{a}$ by $\bar{Q}=\left[\begin{array}{cc}I & 0 \\ -\Gamma & I\end{array}\right]$, and $\bar{Q}^{-1}=\left[\begin{array}{cc}I & 0 \\ \Gamma & I\end{array}\right]$, respectively, and by conditions (29) and (30), one can obtain

$$
\bar{Q} \bar{A}_{a} \bar{Q}^{-1}=\left[\begin{array}{cc}
A_{f}-B_{f} K & -B_{f} K Q_{2} \\
0 & A_{z}-H C_{z}+\Gamma B_{f} K Q_{2}
\end{array}\right] .
$$

Therefore, $\quad \operatorname{eig}\left\{\bar{A}_{a}\right\}=\operatorname{eig}\left\{A_{f}-B_{f} K\right\} \quad \cup$ eig $\left\{A_{z}-H C_{z}+\Gamma B_{f} K Q_{2}\right\}$, where eig $\{*\}$ denotes the eigenvalues of matrix $*$. The matrix $\left(A_{z}-H C_{z}+\Gamma B_{f} K Q_{2}\right)$ is negative definite if conditions of Proposition 1 are satisfied. The characteristic equation of the matrix $A_{f}-B_{f} K$ can be expressed as

$$
\operatorname{det}\left(S I_{3}-\left(A_{f}-B_{f} K\right)\right)=S^{3}+\frac{k_{3}}{\tau_{i}} S^{2}+\frac{k_{2}}{\tau_{i}} S+\frac{k_{1}}{\tau_{i}} .
$$

According to Routh-Hurwitz stability criterion [38], this polynomial (35) is Hurwitz stable under condition (31). This implies that all eigenvalues of $\bar{A}_{a}$ and consequently $A_{a}$ have negative real parts.

The conditions for the platoon asymptotic stability can be established by the following theorem.

Theorem 1. Consider the cooperative control of a platoon of connected car-like vehicles with dynamics defined in (2), (3) - (9), and (11), with assumptions 1 - 5, and the observerbased control algorithm proposed in (15) and (16) is applied to it. Then, the system is asymptotically stable as long as the
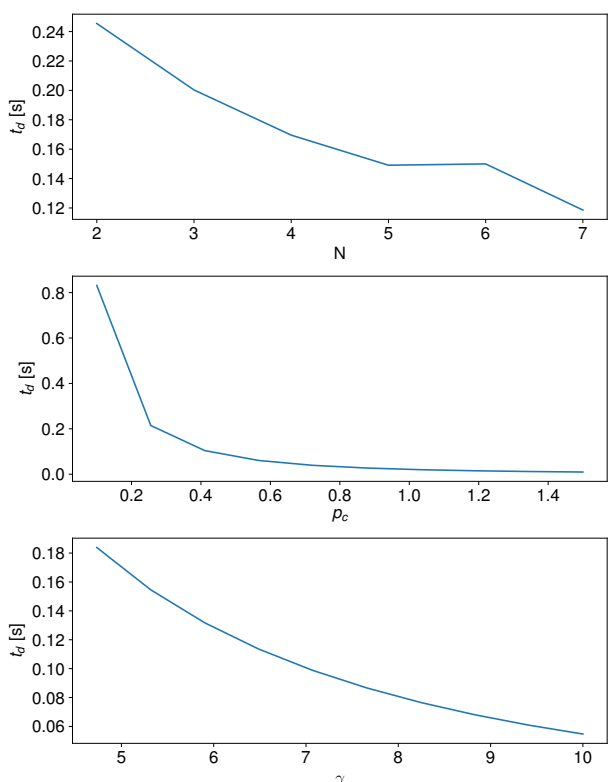

Figure 5. Influence of $\mathrm{N}$ (top), $p_{c}$ (middle) and $\gamma$ (bottom) on the upper bond $t_{d}$ for the time delay. Unless varying, parameters are chosen as $N=$ $3, p_{c}=0.3, \gamma=5$.

conditions (29) - (32) given in Proposition 1 are met and the upper bound of time delay is given by

$$
t_{d}<\frac{\|\Lambda-Q\|}{\left\|P A_{m} P^{-1} A_{m}^{T} P+\frac{1}{2} P+P A_{d}^{2} P^{-1}\left(A_{d}^{2}\right)^{T} P\right\|}
$$

The proof depends mainly on the well-known LyapunovKrasovskii theorem and is detailed in Appendix A.

Remark 2. To get a unique solution to Sylvester equation (29), $\Gamma$, the matrices $\left(A_{z}-H C_{z}\right)$ and $\left(A_{f}-B_{f} K\right)$ have to be designed such that there are no eigenvalues in common between them.

Remark 3. Adding observer to the control loop increases the complexity of the string stability analysis. Therefore, the next corollary is proposed in order to reduce the required design parameters and to simplify the observer-based control design and its string stability analysis.

Remark 4. While (36) is highly non-linear and depends on the dynamics of the system, a numerical example of time delay upper bound is shown in Fig.5. As expected, the more challenging the configuration (higher $N, p_{c}$ or $\gamma$ ) the smaller $t_{d}$ limitation.

Corollary 1. The proposed platoon observer-based control system is asymptotically stable provided that condition (36) is 
satisfied and the design parameters are selected as

$$
\begin{aligned}
k_{1} & =\tau_{i} p_{c}^{3}, \\
k_{2} & =3 \tau_{i} p_{c}^{2}, \\
k_{3} & =3 \tau_{i} p_{c}, \\
h_{1} & =2 p_{o}, \\
h_{2} & =p_{o}^{2}, \\
p_{o} & =\gamma p_{c}, \forall \gamma, p_{c}, \text { and } p_{o}>0, \\
\gamma & \geq \frac{71}{15},
\end{aligned}
$$

where the desired eigenvalues of controller part $\left(A_{f}-B_{f} K\right)$ are assigned at $-p_{c}$, and that of the observer part, $\left(A_{z}-\right.$ $\left.H C_{z}\right)$, are placed at $-p_{o} . \gamma$ is a constant that determines the distance between the poles of the controller and the observer.

Proof: By choosing $K$ and $H$ as given in conditions (37a) - (37e), all the poles of $\left(A_{f}-B_{f} K\right)$ and $\left(A_{z}-H C_{z}\right)$ are placed at $-p_{c}$ and $-p_{o}$ respectively. Hence, one can choose the eigenvalues to be different by satisfying ( $37 \mathrm{~g})$. Thus, according to Remark 2, solution of (29), $\Gamma$, exists, and consequently, condition (30) can be met.

Checking stability conditions of (35), and setting the value of $k_{1}, k_{2}$, and $k_{3}$, as given in (37a) - (37c), we can satisfy condition (31).

Substitution (37a) - (37f) into (32), after some algebraic and numeric manipulations, one can get a condition for $\gamma$ as given in $(37 \mathrm{~g})$.

Remark 5. In practice, tuning the parameter $\gamma$ can be done by starting from the exposed lower bound and increase it as long as the induced behavior does not get too sensitive to measurement noise. The optimal value for $\gamma$ thus depends on the desired behavior.

\section{String Stability}

In addition to asymptotic stability, the platoon controller has to guarantee string stability, including a leading vehicle with index $i=0$ and $N$ following vehicles with index $i=$ $1,2, \ldots, N$. String stability prevents the error signals from amplifying when propagating downstream the vehicle string, which may lead to rear-end collision.

Definition 1. $L_{2}$ String Stability means that the energy (represented by the $L_{2}$ norm) of the position error of vehicle $i, e_{s, i}$, is smaller than the energy of the position error of preceding vehicle $j=i-1, e_{s, j}$ [39]. Since $\left\|\bar{G}(S)=\frac{E_{i}(S)}{E_{j}(S)}\right\|_{\infty}=$ $\sup _{e_{s, j} \in L_{2}}\left\|e_{s, i}\right\|_{2}$, the string stability is guaranteed if

$$
\|\bar{G}(s)\|_{\infty}<1 .
$$

For notation and analysis simplicity, let us define $e_{i}(t)=$ $s_{j}(t)-s_{i}(t)-d_{r, i}, \dot{e}_{i}(t)=q_{j}(t)-q_{i}(t), e_{i 0}(t)=s_{0}(t)-$ $s_{i}(t)-d_{r, i 0}, \dot{e}_{i 0}(t)=q_{0}(t)-q_{i}(t)$, and $\ddot{e}_{i}(t)=\eta_{j}(t)-\eta_{i}(t)$.

Assumption 6. All the vehicles have equal dynamics, i.e., $\tau_{i}=\tau \forall i=1,2, \ldots, N$.
Theorem 2. Consider the platoon system presented in Theorem 1, with assumptions $1-6$, and the observer-based control algorithm proposed in (16) and its parameterization given in Corollary 1. Then, the platoon is string stable as long as the Corollary 1 conditions are satisfied, the time delay $t_{d}$ is upper bounded by a functional of the observer parameters, and the choice of factor $\gamma$ and pole $p_{c}$ is constrained by

$$
\rho \geq \frac{11}{2} \sqrt{p_{c}} .
$$

The proof is given in Appendix B. In particular, the upper bound for $t_{d}$ is given in (68) and (70). This implies that given an estimation of the time delay, suitable observer and control poles can be chosen in order to keep the overall loop stable and string-stable.

After studying of the asymptotic and string stability, the rest of this paper tackles the validation of our theoretical results.

\section{RESUlTS AND Discussions}

In this section, we present both simulation and real-time experiments.

\section{A. Study Setup}

To emulate a realistic setup, the proposed control strategy is tested by implementing the car-like vehicles platoon in a vehicular mobility simulator called ICARS that is developed under the well-known Robot Operating System (ROS). ICARS has features such as real vehicle dynamics and models (engine, transmission, braking systems, etc.), both low and high level measurement units with noises, low level communication module between different parts of the vehicle and the on-board computer, real maps of an urban environment with different road conditions (curvature, bumps, etc.), vehicle communication modules, fully compatible with embedded computer architecture to test real platoon experiments, and HumanMachine Interface to send high-level commands to the vehicle in either simulation or real-time.

We consider a platoon composed of $N$ follower vehicles (of type Robotized Renault ZOE-Q90 LIFE) plus a manually driven leader (of type Renault FLUENCE electric). The parameters of the vehicles are given in Table I. Furthermore, the parameter $\tau$ is estimated by experimental tests. For that a real-time input-output data is recorded and the low-level model of the vehicle, (5), is estimated by using the MATLAB Identification Toolbox. The platoon is tested in the Ecole Centrale de Nantes (ECN) campus as an example of urban environment (road with curvatures, bumps, humans, etc.). The maps of ECN are shared offline among the vehicles. As the leader moves from its starting position to the destination points, its path is constructed online and sent by the radio communication module to the followers as the reference path. The radio system receives the vehicle information in the platoon one by one. Thus, a synchronization algorithm is developed in the ICARS framework to receive and synchronize the information from the radio module.

The lateral controller presented in [32] produces the steering angle of the vehicle to guide the cars to follow the reference 
Table I

Physical Vehicles Parameters

\begin{tabular}{|c|c|c|}
\hline Parameter & VLehicle & \multirow{2}{*}{ ZLE } \\
\hline Mass & $1605 \mathrm{~kg}$ & $1428 \mathrm{~kg}$ \\
\hline Width & $1.545 \mathrm{~m}$ & $1.945 \mathrm{~m}$ \\
\hline Height & $1.462 \mathrm{~m}$ & $1.562 \mathrm{~m}$ \\
\hline Length & $4.748 \mathrm{~m}$ & $4.084 \mathrm{~m}$ \\
\hline Wheelbase & $1.140 \mathrm{~m}$ & $0.657 \mathrm{~m}$ \\
\hline distance between axes & $2.701 \mathrm{~m}$ & $2.588 \mathrm{~m}$ \\
\hline distance between wheels & $1.545 \mathrm{~m}$ & $1.511 \mathrm{~m}$ \\
\hline Wheel radius & $0.29 \mathrm{~m}$ & $0.29 \mathrm{~m}$ \\
\hline Inertia momentum & $45.0 \mathrm{kgm}^{2}$ & $28.0 \mathrm{kgm}^{2}$ \\
\hline$\tau$ & - & 0.2 \\
\hline
\end{tabular}

path of the leader (by minimizing both the lateral and angular deviations, $r_{i}$ and $\psi_{i}$, respectively).

The longitudinal controller runs at a rate of $100 \mathrm{~Hz}$. Control parameters are selected to guarantee the asymptotic stability and string stability given in Theorem 1, Corollary 1 and Theorem 2. Table II presented the control parameters. In addition, by using this setting of parameters, the theoretical upper bound of time delay, satisfying the conditions provided in (36), (68) and (70), is $44.9235 \mathrm{~ms}$. For safety and comfortability, the vehicle velocity and acceleration are constrained to certain levels as given in Table II. Simulation and real-time

Table II

CONTROLLER PARAMETERS FOR SIMULATION AND EXPERIMENTS

\begin{tabular}{|c|c|c|c|}
\hline Parameter & Value & Parameter & Value \\
\hline$u_{i}$ & $\in[-6,1] \mathrm{m} / \mathrm{s}^{2}$ & $v_{i}$ & $\in[0,8] \mathrm{m} / \mathrm{s}$ \\
\hline$d_{r, i}$ & $10 \mathrm{~m}$ & $\gamma$ & 6 \\
\hline$p_{c}$ & 1 & $\Lambda$ & $10000 I_{5 N}$ \\
\hline$Q$ & $0.0001 I_{5 N}$ & & \\
\hline
\end{tabular}

experiments are demonstrated in the video attached to this article $^{2}$.

\section{B. Simulation Study}

In order to illustrate the string stability and the estimation errors well, a three ZOE follower vehicles (i.e., $N=3$ ) are used in a prior simulation studies.

Results of the lateral motion are illustrated in Fig. 6a. The reference and actual paths of the leader and followers respectively are shown in Fig. 6a. Fig. 6b presents the time history of lateral deviation of the followers with respect to the reference path generated form the leader. During the platoon initialization, it is noted higher deviations, due to the time taken by the followers to join the platoon and converge to the leader path, which is gradually attenuated. The deviations increase when the leader and followers move through a curved segmented of the road. These figures enlighten the tracking capabilities of the lateral controller.

Figures $6 c-6 f$ show the longitudinal motion results. As shown in Fig. 6c, the leader moves with variable velocity depending on the path conditions (manually by a human

\footnotetext{
${ }^{2}$ https://box.ec-nantes.fr:443/index.php/s/wtBB3E4YRabnpTN
}

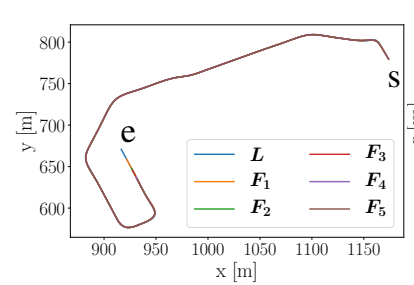

(a) Path

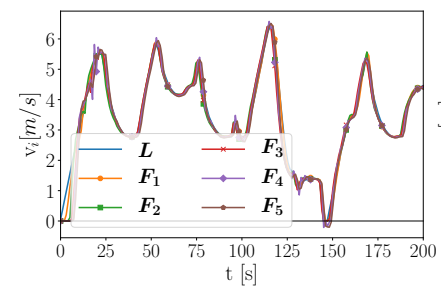

(c) Vehicles move with variable velocity

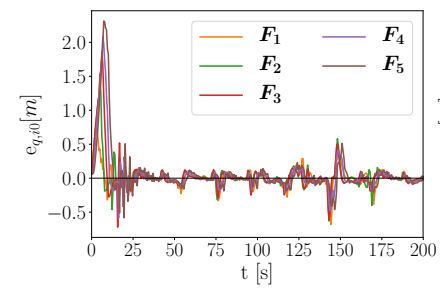

(e) Velocity tracking

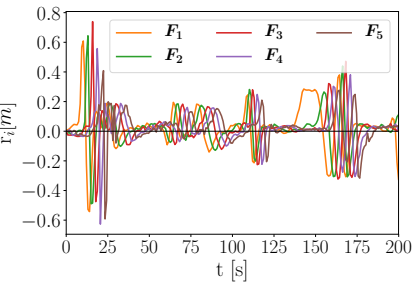

(b) Lateral error

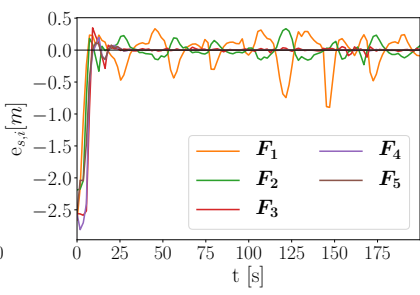

(d) Position tracking

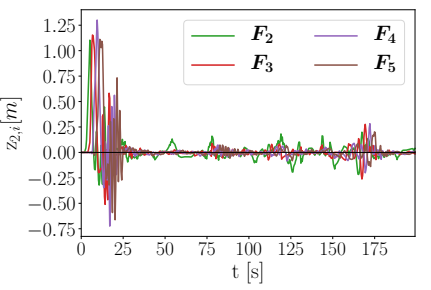

(f) $\tilde{z}_{2, i}=e_{q, i j}-\hat{z}_{2, i}$
Figure 6. Platoon simulation results. $L$ : leader actual path, and $F_{i}$ : follower $i$ actual path. The followers track the path perfectly

driver) which reflects the nature of navigation in urban environments. The vehicles start from distances different from the desired inter-vehicle distance and reach the consensus where the curvilinear position and velocity tracking errors converge. At certain places in the path (e.g., road curvature), the position and velocity errors become higher. These are desired to allow the followers to adapt their velocity based on the road conditions independently from the leader velocity. History of the observer estimation error, $\tilde{z}_{2, i}=e_{q, i j}-\hat{z}_{2, i}$, is given Fig. 6f which shows convergence of the estimated predecessor velocity.

To quantify our results, the Root Mean Squared Error (RMSE) of the tracking and estimation errors, calculated after the passing of an initialization period, are given in Table III which are in the acceptable ranges. Furthermore, this table shows that the position tracking errors are attenuated as the RMSE of $e_{s, i+1}<e_{s, i}$, which enlightens the string stability of the platoon.

\section{Experimental Results}

We have two real following vehicles of type ZOE, so $N=2$ in the real-time experiments, see Fig. 7. Each vehicle has an intelligent sensing module including a set of navigation sensors and localization algorithms to determine the vehicle actual status, e.g., position, velocity, acceleration, heading, etc. In addition, each of them is equipped with a radio module (ARM-N8-SIGFOX with $868 \mathrm{Mhz} 1 / 2$ wave antenna), 
Table III

RMSE OF TRACKING AND ESTIMATION ERRORS IN SIMULATION.

\begin{tabular}{|c|c|c|c|c|c|}
\hline RMSE $\quad$ Follower $i$ & 1 & 2 & 3 & 4 & 5 \\
\hline$e_{s, i}[m]$ & 0.267 & 0.114 & 0.029 & 0.023 & 0.019 \\
\hline$e_{q, i}[\mathrm{~m} / \mathrm{s}]$ & 0.119 & 0.063 & 0.048 & 0.039 & 0.033 \\
\hline$\tilde{z}_{2, i}[\mathrm{~m} / \mathrm{s}]$ & - & 0.063 & 0.048 & 0.039 & 0.033 \\
\hline$r_{i}[m]$ & 0.132 & 0.108 & 0.110 & 0.111 & 0.105 \\
\hline
\end{tabular}

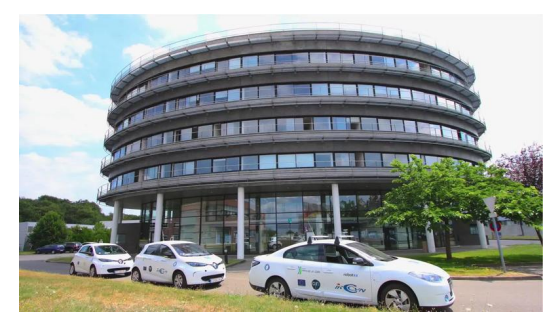

Figure 7. Platoon prototype in the Ecole Centrale de Nantes campus.

an IMU (XSENS MTI-100), a GPS-RTK (PROFLEX-800), a laser ranger (Velodyne VLP-16), and an Onboard Computer (Doliath 1000 - Intel Core i5-3610ME 2.10GHz). The software architecture is developed under ROS Kinetic on Ubuntu 16.04 LTS-64 bits. Expected communication is performed at $10 \mathrm{~Hz}$ in order to cope with packet loss. On each vehicle, the received data is processed by an embedded Kalman Filter in order to estimate the state of its precedessors (including the leading vehicle) at a higher rate.

A study similar to that done in simulation is implemented experimentally. Figures 8a, 8b show the experimental results of path tracking and lateral deviations which illustrate the tracking capabilities of the lateral controller.

Figures $8 c-8 f$ present the longitudinal motion results in real-time. The vehicles start from distances different from the desired inter-vehicle distance and reach the consensus where the curvilinear position tracking, velocity tracking and front vehicle velocity estimation errors converge considering the road conditions. The experimental results ensure the capability of the proposed approach of creating and maintaining the platoon in case of a manually driven leader (see Fig. 8c). Table IV gives the RMSE of the tracking and estimation errors which are larger than that obtained in the simulation study but they are still in the acceptable ranges. Moreover, it indicates that the position tracking errors are attenuated as the RMSE of $e_{s, 2}<e_{s, 1}$, which validates the string stability of the platoon in real-time.

\section{CONClusion}

A successful application of an observer-based longitudinal controller for car-like vehicles platoon navigating in an urban environment is presented. Platoon kinematic and dynamic models are introduced in both curvilinear and Cartesian coordinates with a framework that allows designing the control law in the curvilinear coordinates considering the actuator dynamics. An observer-based control law is proposed to achieve both vehicles consensus and string stability. This

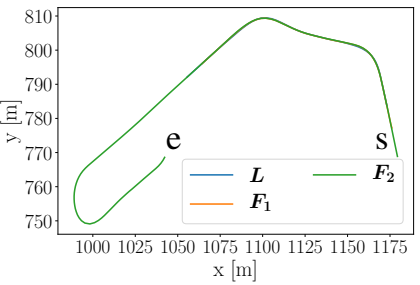

(a) Path tracking: path

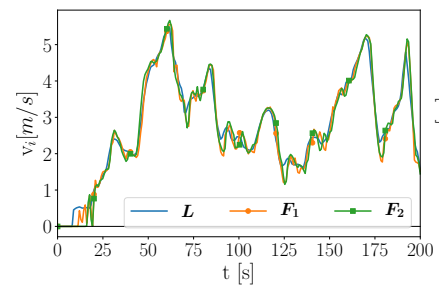

(c) Vehicles travel with variable velocity

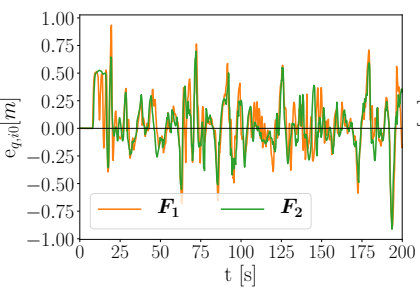

(e) Velocity tracking

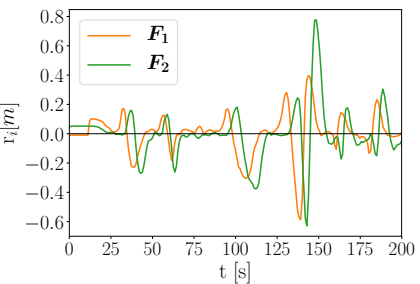

(b) Path tracking: lateral error

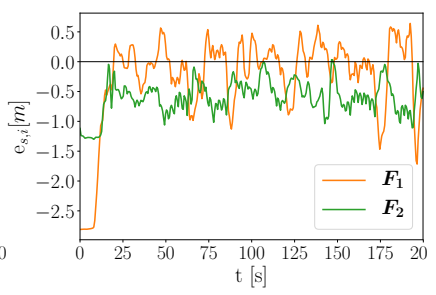

(d) Position tracking

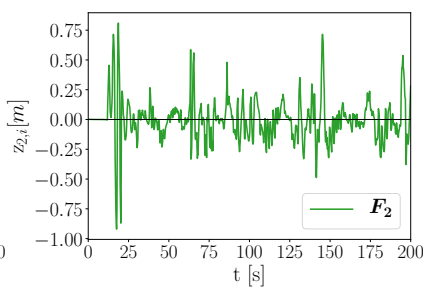

(f) Velocity estimation error

Figure 8. Platoon experimental results.

Table IV

RMSE OF TRACKING ERRORS IN REAL-TIME

\begin{tabular}{|c|c|c|}
\hline FMSE Follower $i$ & 1 & 2 \\
\hline$e_{s, i}[\mathrm{~m}]$ & 0.5623 & 0.4876 \\
\hline$e_{q, i}[\mathrm{~m} / \mathrm{s}]$ & 0.2360 & 0.1317 \\
\hline$\tilde{z}_{2, i}[\mathrm{~m} / \mathrm{s}]$ & - & 0.1317 \\
\hline$r_{i}[\mathrm{~m}]$ & 0.1488 & 0.1339 \\
\hline$\psi_{i}[\mathrm{rad}]$ & 0.0300 & 0.0179 \\
\hline
\end{tabular}

control scheme requires a minimum number of communication links meanwhile it can achieve a high traffic capacity by utilization of information from sensor-based and communicationbased links. The missing predecessor velocity information is estimated by an observer. Compared to configurations where the predecessor velocity can be directly measured, the main limit is that the inter-vehicle distance should be increase in order to account for increased time delays and convergence of the observer. Conditions for platoon asymptotic stability and string stability are provided considering time delay. Both simulation and real-time studies are conducted to enlighten the efficiency of the proposed approach.

\section{ACKNOWLEDGMENT}

The work demonstrated in this paper is carried out in the framework of the Valet project, reference ANR-15-CE220013-02. Parts of the equipment used here were funded by 
the project ROBOTEX, reference ANR-10-EQPX-44-01. Furthermore, we would like to express our gratitude to both Prof. Gaetan Garcia and Dr. Arnaud Hamon, from Ecole Centrale de Nantes, for their efforts and fruitful discussions during the experiments.

\section{APPENDIX}

\section{A. Proof of Theorem 1}

Lemma 1. (Lyapunov-Krasovskii Stability Theorem [40]). Let $\phi(\delta) \in \mathcal{C}[a, b] \rightarrow R^{n}$ be the set of continuous functions mapping the interval $[a, b]$ to $\mathbb{R}^{n}$, then the uniform norm of $\phi$ is defined as

$$
\|\phi\|_{c}=\max _{a \leq \delta \leq b}\|\phi(\delta)\|
$$

Given a system of the form

$$
\begin{aligned}
\dot{\bar{x}}(t) & =g(t, \bar{x}(t)), t \geq t_{0}, \\
\bar{x}\left(t_{0}+\delta\right) & =\phi(\delta), \forall \delta \in[-\beta, 0],
\end{aligned}
$$

where $\beta>0$ is the delay and $\phi \in \mathcal{C}[-\beta, 0] \rightarrow \mathbb{R}^{n}$ is the function of initial conditions. Then, the following result holds: Suppose that the function $g: \mathbb{R} \times \mathcal{C}[-\beta, 0] \rightarrow \mathbb{R}^{n}$ maps $\mathbb{R} \times$ (bounded sets in $\mathcal{C}[-\beta, 0]$ ) into bounded sets of $\mathbb{R}^{n}$. Let $\sigma_{1}, \sigma_{2}$, $\sigma_{3}: \mathbb{R}_{+} \rightarrow \mathbb{R}_{+}$be continuous nondecreasing functions with $\sigma_{1}(\delta)>0$ and $\sigma_{2}(\delta)>0$ for $\delta>0$ and $\sigma_{1}(0)=\sigma_{2}(0)=0$. Assume further there exists a continuous differential function $V: \mathbb{R} \times \mathcal{C}[-\beta, 0] \rightarrow \mathbb{R}^{n}$ such that

$$
\sigma_{1}(\|\phi(0)\|) \leq V(t, \phi) \leq \sigma_{2}\left(\|\phi\|_{c}\right),
$$

and its derivative along (40) is non-positive in the sense that

$$
\dot{V}(t, \phi) \leq-\sigma_{3}(\|\phi(0)\|),
$$

then the trivial solution of (40) is uniformly stable. Moreover, if $\sigma_{3}(\delta)>0$ for $\delta>0$, then it is uniformly asymptotically stable. In addition, if $\lim _{\delta \rightarrow \infty} \sigma_{1}(\delta)=+\infty$, then it is globally uniformly asymptotically stable.

Let $P \in \mathbb{R}^{5 N \times 5 N}$ with $P=P^{T}>0$ and $\Lambda \in \mathbb{R}^{5 N \times 5 N}$ with $\Lambda>0$ are appropriately chosen matrices, and consider the following Lyapunov-Krasovskii function for the system in (27)

$$
V(t)=X(t)^{T} P X(t)+\int_{t-t_{d}}^{t} X(\xi)^{T} \Lambda X(\xi) d \xi .
$$

According to the hypotheses of Lemma 1, let us define the following continuous nondecreasing and positive functions as

$$
\begin{gathered}
\sigma_{1}(X(t))=X(t)^{T} P X(t) \\
\sigma_{2}\left(X\left(t-t_{d}\right)\right)=X(t)^{T} P X(t)+\int_{t-t_{d}}^{t} X(\xi)^{T} \Lambda X(\xi) d \xi
\end{gathered}
$$

From definitions in (43) and (44), condition (41) is satisfied. Differentiating the function in (43) yields

$$
\dot{V}(t)=\dot{X}(t)^{T} P X(t)+X(t)^{T} P \dot{X}(t)+\left.\left(X(\xi)^{T} \Lambda X(\xi) \dot{\xi}\right)\right|_{\substack{\xi=t-t_{d} \\(45)}} ^{\xi=t}
$$

Substituting (27) into (45), after some algebraic manipulations, we have

$$
\begin{aligned}
\dot{V}(t)= & X(t)^{T}\left(P A_{a}+A_{a}^{T} P\right) X(t) \\
& +X(t)^{T} \Lambda X(t)-X\left(t-t_{d}\right)^{T} \Lambda X\left(t-t_{d}\right) \\
& -2 X(t)^{T} P A_{m} \int_{-t_{d}}^{0} x(t+\rho) d \rho \\
& -2 X(t)^{T} P A_{d}^{2} \int_{-t_{d}}^{0} x\left(t+\rho-t_{d}\right) d \rho .
\end{aligned}
$$

From Proposition 1, the matrix $A_{a}$ is Hurwitz stable, and hence from Lyapunov theorem, we have

$$
P A_{a}+A_{a}^{T} P=-Q,
$$

where $Q=Q^{T}>0$.

Lemma 2. [41]. For any positive definite matrix $\mathcal{W}$ with suitable dimensions, it holds

$$
2 a^{T} c \leq a^{T} \mathcal{W} a+c^{T} \mathcal{W}^{-1} c
$$

According to Lemma 2, choosing $a=-X^{T} P A_{m}, c=$ $x(t+\rho), \mathcal{W}=P^{-1}$, and integrating both side of the inequality, we have

$$
\begin{aligned}
& 2 X(t)^{T} P A_{m} \int_{-t_{d}}^{0} x(t+\rho) d \rho \\
& \leq t_{d} X(t)^{T} P A_{m} P^{-1} A_{m}^{T} P X(t) \\
& \quad+\int_{-t_{d}}^{0} X(t+\rho)^{T} P X(t+\rho) d \rho .
\end{aligned}
$$

Similarly, one can obtain

$$
\begin{aligned}
& 2 X(t)^{T} P A_{d}^{2} \int_{-t_{d}}^{0} x\left(t+\rho-t_{d}\right) d \rho \\
& \leq t_{d} X(t)^{T} P A_{d}^{2} P^{-1}\left(A_{d}^{2}\right)^{T} P X(t) \\
& \quad+\int_{-t_{d}}^{0} X\left(t+\rho-t_{d}\right)^{T} P X\left(t+\rho-t_{d}\right) d \rho .
\end{aligned}
$$

Lemma 3. [42]. Let $g: \mathcal{C} \subseteq \mathbb{R} \rightarrow \mathbb{R}$ be a convex mapping defined on the interval $\mathcal{C}$ of real numbers, then the following inequality holds

$$
\frac{1}{b-a} \int_{a}^{b} g(\bar{x}) d \bar{x} \leq \frac{g(a)+g(b)}{2},
$$

where $a, b \in \mathcal{C}$ and $a<b$.

Exploiting Lemma 3 for the integral expressions in (49) and (50), it yields

$$
\begin{aligned}
& 2 X(t)^{T} P A_{m} \int_{-t_{d}}^{0} x(t+\rho) d \rho \\
& <t_{d} X(t)^{T} P A_{m} P^{-1} A_{m}^{T} P X(t) \\
& \quad+\frac{t_{d}}{2}\left(X(t)^{T} P X(t)+X\left(t-t_{d}\right)^{T} P X\left(t-t_{d}\right)\right)
\end{aligned}
$$


and

$$
\begin{aligned}
2 X(t)^{T} P A_{d}^{2} \int_{-t_{d}}^{0} x\left(t+\rho-t_{d}\right) d \rho \\
\leq t_{d} X(t)^{T} P A_{d}^{2} P^{-1}\left(A_{d}^{2}\right)^{T} P X(t)+ \\
\quad+\frac{t_{d}}{2}\left(X\left(t-t_{d}\right)^{T} P X\left(t-t_{d}\right)+X\left(t-2 t_{d}\right)^{T} P X\left(t-2 t_{d}\right)\right) .
\end{aligned}
$$

Substituting (47), (52) and (53) into (46), after some algebraic manipulations, we have

$$
\begin{aligned}
\dot{V}< & X(t)^{T}\left(-Q+\Lambda-t_{d} P A_{m} P^{-1} A_{m}^{T} P-\frac{t_{d}}{2} P\right. \\
& \left.-t_{d} P A_{d}^{2} P^{-1}\left(A_{d}^{2}\right)^{T} P\right) X(t) \\
+ & X\left(t-t_{d}\right)^{T}\left(-\Lambda-t_{d} P\right) X\left(t-t_{d}\right) \\
+ & X\left(t-2 t_{d}\right)^{T}\left(-\frac{t_{d}}{2} P\right) X\left(t-2 t_{d}\right) .
\end{aligned}
$$

Suppose $\zeta=\left[X(t), X\left(t-t_{d}\right), X\left(t-2 t_{d}\right)\right]^{T}$, then (54) can be recast in a compact form as

$$
\dot{V}=\zeta^{T} \Delta \zeta,
$$

where $\Delta=\operatorname{blkdiag}\left\{\Delta_{1}, \Delta_{2}, \Delta_{3}\right\}$, with $\Delta_{1}=-Q+\Lambda-$ $t_{d} P A_{m} P^{-1} A_{m}^{T} P-\frac{t_{d}}{2} P-t_{d} P A_{d}^{2} P^{-1}\left(A_{d}^{2}\right)^{T} P, \Delta_{2}=-\Lambda-$ $t_{d} P$ and $\Delta_{3}=\frac{t_{d}}{2} P$.

According to Lemma 1 and condition (42), to guarantee the uniform stability, we have to prove that $\Delta<0$. Exploiting that $\Lambda>0$ and $P>0$, then $\Delta_{2}<0$ and $\Delta_{3}<0$. Consequently, $\Delta<0$ if $\Delta_{1}<0$, i.e., by satisfying condition (36).

Now, according to Lemma 1 , given the selected form of $\sigma_{1}(X(t))$ in (44), the delayed closed-loop system (23) is also globally asymptotically stable.

\section{B. Proof of Theorem 2}

Let us start with

$$
\dddot{e}_{i}(t)=\dot{\eta}_{j}(t)-\dot{\eta}_{i}(t) .
$$

Substituting (11) and (16) into (56), considering the relations $e_{i}=e_{i 0}-e_{j 0}$ and $\dot{e}_{i}=\dot{e}_{i 0}-\dot{e}_{j 0}$, and after some algebraic manipulations, we get

$$
\begin{aligned}
\tau \dddot{e}_{i}(t)= & -g_{c, 3} \ddot{e}_{i}(t)-g_{c, 2} \dot{e}_{i}\left(t-t_{d}\right)-g_{c, 1} e_{i}\left(t-t_{d}\right) \\
& -g_{o, 1} \hat{z}_{1, i}(t)-g_{o, 2} \hat{z}_{2, i}(t)+g_{o, 1} \hat{z}_{1, j}(t)+g_{o, 2} \hat{z}_{2, j}(t) .
\end{aligned}
$$

The observer model given (19) can be represented in the form of transfer functions as $\bar{G}_{z_{1}}(S)=\frac{\hat{Z}_{1, i}(S)}{E_{j}(S)}$ and $\bar{G}_{z_{2}}(S)=$ $\frac{\hat{Z}_{2, i}(S)}{E_{j}(S)}$.

Expressing (57) in the S-domain, one can obtain

$$
\begin{array}{r}
\bar{G}(S)=\frac{g_{o, 1} \bar{G}_{z_{1}}+g_{o, 2} \bar{G}_{z_{2}}}{\tau S^{3}+g_{c, 3} S^{2}+g_{c, 2} S e^{-t_{d} S}+g_{c, 1} e^{-t_{d} S}} . \\
+g_{o, 1} \bar{G}_{z_{1}}+g_{o, 2} \bar{G}_{z_{2}}
\end{array}
$$

Since $\|\bar{G}(S)\|_{\infty}=\sup _{w>0} \sqrt{|\bar{G}(j w)|^{2}}=\sup _{w>0} \sqrt{\frac{\bar{G}_{n}(w)}{\bar{G}_{d}(w)}}$, string stability criterion (38) is equivalent to

$$
\bar{G}_{d}(\omega)-\bar{G}_{n}(\omega)>0, \forall \omega>0 .
$$

Exploiting the definitions of $G_{c}$ and $G_{o}$ along with (37a) (37f), after certain algebraic manipulations, (59) is recast as

$$
\begin{aligned}
\bar{G}_{d}(\omega)-\bar{G}_{n}(\omega)= & a_{10}^{2} \omega^{10}+\left(a_{8,1} \cos \left(t_{d} \omega\right)+a_{8,2}\right) \omega^{8} \\
& +a_{7} \sin \left(t_{d} \omega\right) \omega^{7} \\
& +\left(a_{6,1} \cos \left(t_{d} \omega\right)+a_{6,2}\right) \omega^{6} \\
& +a_{5} \sin \left(t_{d} \omega\right) \omega^{5} \\
& +\left(a_{4,1} \cos \left(t_{d} \omega\right)+a_{4,2}\right) \omega^{4} \\
& +a_{3} \sin \left(t_{d} \omega\right) \omega^{3} \\
& +\left(a_{2,1} \cos \left(t_{d} \omega\right)+a_{2,2}\right) \omega^{2}+a_{0}
\end{aligned}
$$

where coefficients $a_{(.)}$are functions of $\gamma$ and $p_{c}$.

The following facts can be used to complete the analysis:

$$
\begin{aligned}
& -\sin (\bar{a}) \geq-\bar{a}, \forall \bar{a}>0, \\
& -\cos (\bar{a}) \geq-1, \\
& \bar{a} \sin (\bar{\phi})+\bar{b} \cos (\bar{\phi}) \geq-\sqrt{\bar{a}^{2}+\bar{b}^{2}}, \\
& \sqrt{1+\bar{c}} \approx 1+\frac{1}{2} \bar{c}, \forall \bar{c} \ll 1 .
\end{aligned}
$$

After some algebraic and numeric manipulations, under condition (39), we find

$$
\begin{aligned}
& a_{0}>0 \\
& a_{3}, a_{4,1}, a_{6,1}, a_{7}, a_{8,1}<0
\end{aligned}
$$

By using (61c), we obtain

$$
\begin{gathered}
a_{5} \sin \left(t_{d} \omega\right) \omega^{5}+\left(a_{2,1} \cos \left(t_{d} \omega\right)+a_{2,2}\right) \omega^{2} \\
\geq-\omega^{2} \sqrt{\left(a_{5} \omega^{3}\right)^{2}+a_{2,1}^{2}}+a_{2,2} \omega^{2} .
\end{gathered}
$$

Exploiting the study presented in [43] which stated that the key region of string stability is at the region of low frequencies at which the spacing errors have most of their energy, then at low frequencies, we can use the fact (61d) to simplify the square root in (63) as $\sqrt{\left(a_{5} \omega^{3}\right)^{2}+a_{2,1}^{2}} \approx a_{2,1}\left(\frac{a_{5}^{2} \omega^{6}}{2 a_{2,1}^{2}}+1\right)$. Thus, (63) can be rewritten as

$$
\begin{aligned}
& a_{5} \sin \left(t_{d} \omega\right) \omega^{5}+\left(a_{2,1} \cos \left(t_{d} \omega\right)+a_{2,2}\right) \omega^{2} \gtrsim \\
& -\frac{a_{5}^{2}}{2 a_{2,1}} \omega^{8}+\left(a_{2,2}-a_{2,1}\right) \omega^{2} .
\end{aligned}
$$

Satisfying condition (39), it follows

$$
\left(a_{2,2}-a_{2,1}\right) \omega^{2}>0 .
$$

Exploiting the fact (61a) along with (62b), we obtain

$$
a_{7} \sin \left(t_{d} \omega\right) \omega^{7} \geq-\left|a_{7}\right| t_{d} \omega^{8} .
$$

Gathering the terms of $\omega^{8}$ from (64), (66) and (60), along with (61b) and (62b), and satisfying condition (39), we get

$$
\begin{gathered}
\left(a_{8,1} \cos \left(t_{d} \omega\right)+a_{8,2}-\frac{a_{5}^{2}}{2 a_{2,1}}-\left|a_{7}\right| t_{d}\right) \omega^{8} \\
\geq\left(-\left|a_{8,1}\right|+a_{8,2}-\frac{a_{5}^{2}}{2 a_{2,1}}-\left|a_{7}\right| t_{d}\right) \omega^{8} .
\end{gathered}
$$


Hence, for $\left(-\left|a_{8,1}\right|+a_{8,2}-\frac{a_{5}^{2}}{2 a_{2,1}}-\left|a_{7}\right| t_{d}\right)>0$, along with (39), we set the condition

$$
t_{d}<\frac{-\left|a_{8,1}\right|+a_{8,2}-\frac{a_{5}^{2}}{2 a_{2,1}}}{\left|a_{7}\right|} .
$$

Exploiting the facts (61a) and (61b), it follows

$$
\begin{aligned}
& -a_{3} \sin \left(t_{d} \omega\right) \omega^{3} \geq-\left|a_{3}\right| t_{d} \omega^{4} \\
& \left(a_{4,1} \cos \left(t_{d} \omega\right)+a_{4,2}\right) \omega^{4} \geq\left(-\left|a_{4,1}\right|+a_{4,2}\right) \omega^{4} .
\end{aligned}
$$

Collecting the terms of $\omega^{4}$ in (69), we get the new coefficient of $\omega^{4}$ which is $a_{4,2}-a_{4,1}-\left|a_{3}\right| t_{d}$ that is positive if

$$
t_{d}<\frac{a_{4,2}-\left|a_{4,1}\right|}{\left|a_{3}\right|} .
$$

Meanwhile the condition (39) satisfies that $\left(a_{4,2}-\left|a_{4,1}\right|\right)>0$ that ensures the positiveness of $t_{d}$ upper bound.

Utilizing the fact (61b) with (62b), under condition (39), it follows

$$
\left(a_{6,1} \cos \left(t_{d} \omega\right)+a_{6,2}\right) \omega^{6} \geq\left(-\left|a_{6,1}\right|+a_{6,2}\right) \omega^{6}>0 .
$$

Checking (59), (60), (62a), (64), (65), (67), (69) and (71), then the string stability criteria (59) is guaranteed.

\section{REFERENCES}

[1] J. Bom, B. Thuilot, F. Marmoiton, and P. Martinet, "Nonlinear control for urban vehicles platooning, relying upon a unique kinematic gps," in IEEE Int. Conf. on Robotics and Automation, 2005, pp. 4138-4143.

[2] T. D. Barfoot and C. M. Clark, "Motion planning for formations of mobile robots," Robotics and Autonomous Systems, vol. 46, no. 2, pp. 65-78, 2004.

[3] E. Héry, S. Masi, P. Xu, and P. Bonnifait, "Map-based curvilinear coordinates for autonomous vehicles," in IEEE Intelligent Transportation Systems Conf. IEEE, 2017, pp. 1-7.

[4] S. E. Li, Y. Zheng, K. Li, and J. Wang, "An overview of vehicular platoon control under the four-component framework," in IEEE Intelligent Vehicles Symp., 2015, pp. 286-291.

[5] J.-W. Kwon and D. Chwa, "Adaptive bidirectional platoon control using a coupled sliding mode control method," IEEE Trans. on Intelligent Transportation Systems, vol. 15, no. 5, pp. 2040-2048, 2014.

[6] X. Guo, J. Wang, F. Liao, and R. S. H. Teo, "Neuroadaptive quantized pid sliding-mode control for heterogeneous vehicular platoon with unknown actuator deadzone," Int. Journal of Robust and Nonlinear Control, vol. 29, no. 1, pp. 188-208, 2019.

[7] Y. Ma, Z. Li, R. Malekian, R. Zhang, X. Song, and M. A. Sotelo, "Hierarchical fuzzy logic-based variable structure control for vehicles platooning," IEEE Trans. on Intelligent Transportation Systems, no. 99, pp. 1-12, 2018.

[8] F. Lin, M. Fardad, and M. R. Jovanovic, "Optimal control of vehicular formations with nearest neighbor interactions," IEEE Trans. on Automatic Control, vol. 57, no. 9, pp. 2203-2218, 2012.

[9] Y. Li, C. Tang, S. Peeta, and Y. Wang, "Nonlinear consensus-based connected vehicle platoon control incorporating car-following interactions and heterogeneous time delays," IEEE Trans. on Intelligent Transportation Systems, vol. 20, no. 6, pp. 2209-2219, 2018.

[10] J. C. Zegers, E. Semsar-Kazerooni, J. Ploeg, N. van de Wouw, and H. Nijmeijer, "Consensus control for vehicular platooning with velocity constraints," IEEE Trans. on Control Systems Technology, vol. 26, no. 5, pp. 1592-1605, 2017.

[11] S. E. Li, H. Peng, K. Li, and J. Wang, "Minimum fuel control strategy in automated car-following scenarios," IEEE Trans. on Vehicular Technology, vol. 61, no. 3, pp. 998-1007, 2012.

[12] J. Bom, B. Thuilot, F. Marmoiton, and P. Martinet, "A global control strategy for urban vehicles platooning relying on nonlinear decoupling laws," in IEEE/RSJ Int. Conf. on Intelligent Robots and Systems, 2005, pp. 2875-2880.
[13] P. Avanzini, B. Thuilot, and P. Martinet, "A control strategy taking advantage of inter-vehicle communication for platooning navigation in urban environment," in Int. Workshop on Perception and Navigation For Autonomous Vehicles In Human Environment, 2011.

[14] A. Khalifa, O. Kermorgant, S. Dominguez, and P. Martinet, "Vehicles platooning in urban environment: Consensus-based longitudinal control with limited communications capabilities," in Int. Conf. on Control, Automation, Robotics and Vision, 2018, pp. 809-814.

[15] J. VanderWerf, S. Shladover, N. Kourjanskaia, M. Miller, and H. Krishnan, "Modeling effects of driver control assistance systems on traffic," Transportation Research Record: Journal of The Transportation Research Board, no. 1748, pp. 167-174, 2001.

[16] H. Chehardoli and M. R. Homaeinezhad, "Third-order leader-following consensus protocol of traffic flow formed by cooperative vehicular platoons by considering time delay: constant spacing strategy," The Journal of Systems and Control Engineering, vol. 232, no. 3, pp. 285298, 2018.

[17] M. Fardad, F. Lin, and M. R. Jovanović, "Sparsity-promoting optimal control for a class of distributed systems," in American Control Conf., 2011, pp. 2050-2055.

[18] F. Gao, S. E. Li, Y. Zheng, and D. Kum, "Robust control of heterogeneous vehicular platoon with uncertain dynamics and communication delay," IET Intelligent Transport Systems, vol. 10, no. 7, pp. 503-513, 2016.

[19] Y. Wu, S. E. Li, Y. Zheng, and J. K. Hedrick, "Distributed sliding mode control for multi-vehicle systems with positive definite topologies," in IEEE Conf. on Decision and Control, 2016, pp. 5213-5219.

[20] W. B. Dunbar and D. S. Caveney, "Distributed receding horizon control of vehicle platoons: Stability and string stability," IEEE Trans. on Automatic Control, vol. 57, no. 3, pp. 620-633, 2012.

[21] Y. Zheng, S. E. Li, K. Li, F. Borrelli, and J. K. Hedrick, "Distributed model predictive control for heterogeneous vehicle platoons under unidirectional topologies," IEEE Trans. on Control Systems Technology, vol. 25, no. 3, pp. 899-910, 2016.

[22] S. Santini, A. Salvi, A. S. Valente, A. Pescapé, M. Segata, and R. L. Cigno, "A consensus-based approach for platooning with intervehicular communications and its validation in realistic scenarios," IEEE Trans. on Vehicular Technology, vol. 66, no. 3, pp. 1985-1999, 2017.

[23] M. di Bernardo, A. Salvi, and S. Santini, "Distributed consensus strategy for platooning of vehicles in the presence of time-varying heterogeneous communication delays," IEEE Trans. on Intelligent Transportation Systems, vol. 16, no. 1, pp. 102-112, 2015.

[24] A. Salvi, S. Santini, and A. S. Valente, "Design, analysis and performance evaluation of a third order distributed protocol for platooning in the presence of time-varying delays and switching topologies," Transportation Research Part C: Emerging Technologies, vol. 80, pp. 360-383, 2017.

[25] M. Saeednia and M. Menendez, "A consensus-based algorithm for truck platooning," IEEE Trans. on Intelligent Transportation Systems, vol. 18, no. 2, pp. 404-415, 2017.

[26] A. Khalifa, O. Kermorgant, S. Dominguez, and P. Martinet, "Vehicles platooning in urban environments: Integrated consensus-based longitudinal control with gap closure maneuvering and collision avoidance capabilities," in European Control Conf., 2019, pp. 1695-1701.

[27] D. Swaroop, J. Hedrick, C. Chien, and P. Ioannou, "A comparision of spacing and headway control laws for automatically controlled vehicles," Vehicle System Dynamics, vol. 23, no. 1, pp. 597-625, 1994.

[28] C. Wen, F. Liu, Q. Song, and X. Feng, "Observer-based consensus of second-order multi-agent systems without velocity measurements," Neurocomputing, vol. 179, pp. 298-306, 2016.

[29] L. Gao, B. Xu, J. Li, and H. Zhang, "Distributed reduced-order observerbased approach to consensus problems for linear multi-agent systems,' IET Control Theory \& Applications, vol. 9, no. 5, pp. 784-792, 2015.

[30] D. Ding, Z. Wang, D. W. Ho, and G. Wei, "Observer-based eventtriggering consensus control for multiagent systems with lossy sensors and cyber-attacks," IEEE Trans. on Cybernetics, vol. 47, no. 8, pp. 19361947, 2017.

[31] M. Yan, Y. Tang, P. Yang, and L. Zuo, "Consensus based platoon algorithm for velocity-measurement-absent vehicles with actuator saturation," Journal of Advanced Transportation, vol. 2017, 2017.

[32] B. Thuilot, J. Bom, F. Marmoiton, and P. Martinet, "Accurate automatic guidance of an urban electric vehicle relying on a kinematic gps sensor," IFAC Proceedings Volumes, vol. 37, no. 8, pp. 155-160, 2004.

[33] H. Wang, J. Kearney, and K. Atkinson, "Robust and efficient computation of the closest point on a spline curve," in Int. Conf. on Curves and Surfaces, 2002, pp. 397-406. 
[34] C. Altafini, "Path following with reduced off-tracking for multibody wheeled vehicles," IEEE Trans. on Control Systems Technology, vol. 11, no. 4, pp. 598-605, 2003.

[35] H. K. Khalil, Nonlinear control. Pearson Higher Ed, 2014.

[36] L. Zhang and G. Orosz, "Beyond-line-of-sight identification by using vehicle-to-vehicle communication," IEEE Trans. on Intelligent Transportation Systems, vol. 19, no. 6, pp. 1962-1972, 2017.

[37] S. Darbha, "String stability of interconnected systems: An application to platooning in automated highway systems," IEEE Trans. on Automatic Control, vol. 56, no. 4, pp. 923-929, 1994,

[38] R. C. Dorf and R. H. Bishop, Modern control systems. Pearson, 2011.

[39] J. Eyre, D. Yanakiev, and I. Kanellakopoulos, "A simplified framework for string stability analysis of automated vehicles ," Vehicle System Dynamics, vol. 30, no. 5, pp. 375-405, 1998.

[40] K. Gu, J. Chen, and V. L. Kharitonov, Stability of time-delay systems. Springer Science \& Business Media, 2003.

[41] R. A. Horn and C. R. Johnson, Matrix analysis. Cambridge university press, 1990

[42] J. Hadamard, "Étude sur les propriétés des fonctions entières et en particulier d'une fonction considérée par riemann," Journal De Mathématiques Pures et Appliquées, pp. 171-216, 1893.

[43] P. Seiler, A. Pant, and K. Hedrick, "Disturbance propagation in vehicle strings," IEEE Trans. on Automatic Control, vol. 49, no. 10, pp. 18351842, 2004.

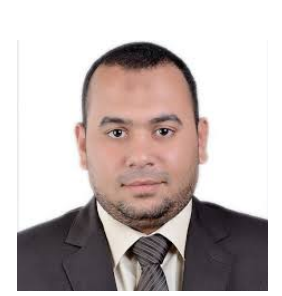

Ahmed Khalifa graduated from Menoufia University in 2009. He has received the M.Sc. and Ph.D. degrees in Mechatronics and Robotics Engineering from Egypt-Japan University of Science and Technology in 2013 and 2016 respectively. In 2015, he joined Namerikawa Laboratory at Keio University as a Ph.D. student. After that, he was recruited as a lecturer of automation and robotics at the Faculty of Electronic Engineering. He is currently a postdoctoral researcher at Ecole Centrale de Nantes. His research interests include mechatronics design and analysis of robotic systems, robust, predictive, cooperative, and distributed control, and their applications in Aerial/Ground Manipulators and Multi-robot systems.

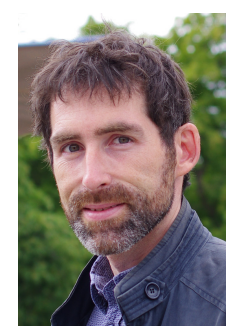

Olivier Kermorgant graduated from École Centrale Paris in 2004. From 2008 to 2011 he was with the Lagadic group at Inria Rennes where he received the $\mathrm{Ph} . \mathrm{D}$. degree in signal processing from University of Rennes in 2011. He then joined the Ocean Systems Lab at Heriot-Watt University, Edinburgh, as a Research Assistant. He was Assistant Professor at University of Strasbourg from 2012 to 2014. Since 2015 he has been Associate Professor at Centrale Nantes. His research interests include sensor-based control, disturbance rejection and optimization.

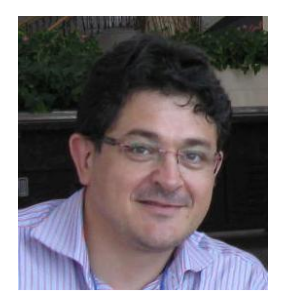

Salvador Dominguez graduated from Universidad de Valladolid, Spain in 1999. From 2000 to 2013 he was with Cartif research center Foundation at Valladolid and received the Ph.D. degree in Social Robotics from University of Valladolid, Spain in 2007. He was granted with an intership at Fraunhofer Research Institute IAIS in Sankt Augustin, Germany in 2011. In 2013 he joined the former IRCCyN now called LS2N in Centrale Nantes, France. His research interests include autonomous navigation in general and self driving cars in particular.

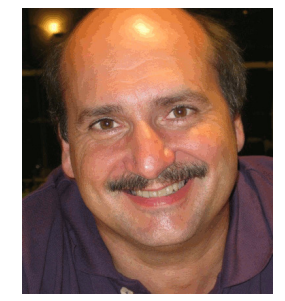

Philippe Martinet graduated from the CUST, Clermont- Ferrand, France, in 1985 and received the $\mathrm{Ph} . \mathrm{D}$. degree in electronics science from the Blaise Pascal University, France, in 1987. From 1990 to 2000, he was assistant Professor with CUST. From 2000 until 2011, he has been a Professor with Institut Français de Mécanique Avancée (IFMA), ClermontFerrand. In 2006, he spent one year as a visiting professor in ISRC at the Sungkyunkwan university in Suwon, South Korea. In September 2011, he moves to Ecole Centrale de Nantes and make his research at LS2N in the robotics team. Until 2015, he stayed associated researcher to Pascal Institute in Clermont-Ferrand. In November 2017, he moves to Inria Sophia Antipolis as Research director. His research interests include visual servoing of robots, multi-sensor-based control, force vision coupling, autonomous guided vehicles, modeling, identification and control of complex machines. From 1990, he is Author and Coauthor of more than three hundred forty references. 